\title{
Investigação da presença de efedrinas em Ephedra tweediana Fisch \& C.A. Meyer e em E. triandra Tul. (Ephedraceae) coletadas em Porto Alegre/RS
}

\author{
Bruna de S. Boff, ${ }^{1,2}$ Viviane C. Sebben, ${ }^{1,2}$ Patrícia K. Paliosa, ${ }^{2}$ Ingrid Azambuja, ${ }^{2}$ Rodrigo B. \\ Singer, ${ }^{3}$ Renata P. Limberger*,2
}

${ }^{1}$ Centro de Informação Toxicológica, Fundação Estadual de Produção e Pesquisa em Saúde do Rio Grande do Sul, Rua Domingos Crescêncio, 132, 90650-090 Porto Alegre-RS, Brasil,

${ }^{2}$ Programa de Pós-Graduação em Ciências Farmacêuticas, Universidade Federal do Rio Grande do Sul, Av. Ipiranga, 2752, 90610-000 Porto Alegre-RS, Brasil,

${ }^{3}$ Departamento de Botânica, Universidade Federal do Rio Grande do Sul, Av. Bento Gonçalves, 9500, Campus do Vale, 91501-970 Porto Alegre-RS, Brasil

\begin{abstract}
RESUMO: Amostras de Ephedra tweediana Fisch \& C.A. Meyer, coletadas de populações nativas da Reserva Biológica do Lami José Lutzenberger (Porto Alegre, RS, Brasil), e amostras de Ephedra triandra Tul., obtidas de plantas cultivadas em Porto Alegre/RS, foram extraídas com acetona, derivatizadas com ciclohexanona e analisadas por CG/EM. Para verificação da eficiência da metodologia, além das amostras de Ephedra tweediana e E. triandra, foram analisadas cinco amostras comerciais de Ephedra, de procedências distintas, cedidas por farmácias de manipulação locais. Os resultados encontrados indicam a ausência de efedrinas em Ephedra tweediana e $E$. triandra e presença de efedrina e/ou pseudoefedrina nas amostras comerciais.
\end{abstract}

Unitermos: Ephedra tweediana, Ephedra triandra, Ephedraceae, efedrina, pseudoefedrina.

\begin{abstract}
Investigation of the presence of ephedrines in Ephedra tweediana Fisch \& C.A. Meyer and E. triandra Tul. (Ephedraceae) collected in Porto Alegre/RS". Samples of Ephedra tweediana, collected from native populations occurring in the Reserva Biológica do Lami José Lutzenberger (Porto Alegre, RS, Brazil), and from cultivated plants of Ephedra triandra were submitted to extraction with acetone, derivatized with cyclohexanone and analyzed by GC/MS. In order to verify the efficiency of the methodology, besides Ephedra tweediana and E. triandra, samples of five commercial Ephedra extracts were analyzed, from distinct origins, get up from local drugstores. The results showed the absence of ephedrines in Ephedra tweediana and E. triandra, and the presence of ephedrine and/or pseudoephedrine in commercial samples.
\end{abstract}

Keywords: Ephedra tweediana, Ephedra triandra, Ephedraceae, ephedrine, pseudoephedrine.

\section{INTRODUÇÃO}

Plantas do gênero Ephedra (Ephedraceae), também conhecidas por "Ma Huang", podem ser encontradas em zonas subtropicais da Ásia, Europa e Américas (WHO, 1999; Soni et al., 2004) e estão listadas na mais antiga matéria médica conhecida, Shen Nong Ben Cao Jing (ABC, 2002). Como um todo, as Ephedraceae são arbustos (raramente arvoretas) muito ramificados, com folhas opostas, escamiformes e muito reduzidas. Os ramos são articulados, com nós e entre-nós muito bem delimitados (Hunziker 1995). Apresentam grande apelo comercial devido à presença de alcalóides derivados da fenilalanina, principalmente $(+)$-pseudoefedrina (isoefedrina ou catina), (+)metilpseudoefedrina, (+)-norpseudonorefedrina, (-)norefedrina, (-)-metilefedrina e (-)-efedrina, sendo este último presente em maiores quantidades (Schaneberg et al., 2003; Barbosa-Filho et al., 2006).

Estudos indicam que existem de 50 a 65 espécies de efedra no mundo (Huang et al., 2005), cuja constituição química varia de acordo com a sua localização geográfica e com as condições edafo climáticas (Tyler, 1993). Espécies da Eurásia são caracterizadas pela presença de alcalóides, enquanto as espécies americanas são citadas por não produzirem tais metabólitos (Foster \& Tyler, 1999; Blumenthal et al., 2000; Schaneberg et al., 2003). Esta característica apresenta dados conflitantes na literatura científica e ainda se questiona se as espécies americanas acumulem efedrinas (Caveney et al., 2001; Schaneberg et al., 2003). Alguns autores relatam presença de alcalóides em espécies de efedra do norte e sul da América (Willaman \& Scheubert, 1961; Wink, 1998). Outros trabalhos demonstram ausência destes metabólitos (Konno et al., 1979; Bruneton, 1992; Caveney et al., 2001) e um perfil 
químico distinto entre extratos obtidos de espécies da Eurásia e de espécies americanas (Schaneberg et al., 2003).

Aproximadamente 40 espécies de efedra são cultivadas para fins comerciais, dentre elas Ephedra sinica Stapf e E. equisetina Bunge, do sul da China, E. intermedia Schrenk. e E. gerardiana Wall., da Índia e do Paquistão, as quais contêm de 0,02 a 3,4\% de alcalóides (Bruneton, 1992; Schaneberg et al., 2003). A composição química destas espécies varia qualitativamente e quantitativamente (Soni et al., 2004). De uma forma geral, efedrina (EF) e pseudoefedrina (PEF) juntas constituem mais de $80 \%$ do total de alcalóides (Gurley et al., 1998).

Segundo a Comissão E (Blumenthal et al., 2002) e a Farmacopéia da República Popular da China (PPRC, 1997), a droga efedra consiste de ramos secos e jovens de E. sinica Stapf, E. shennungiana Tang, ou outras espécies equivalentes do gênero Ephedra, colhidas no outono, época de conteúdo máximo de princípios ativos (Bruneton, 1992). A Farmacopéia Japonesa (JP XIII, 1996) preconiza não menos que $0,7 \%$ de alcalóides totais, a Farmacopéia Chinesa não menos que $0,8 \%$ e a Alemã não menos que 1\% (ABC, 2002), expressos em efedrina e pseudoefedrina (bases anidras).

Preparações a base de Ephedra spp. têm um longo histórico de uso. Na medicina oriental, são denominadas Ma Huang e utilizadas a mais de 5.000 anos como componente de fórmulas multi-ervas prescritas para tratar asma brônquica, gripes, resfriados, tosse, febre, falta de ar, cefaléia e congestão nasal (Foster \& Tyler, 1999; Soni et al., 2004). Na medicina moderna, têm sido usadas no tratamento de doenças do trato respiratório e de broncoespasmos (Blumenthal et al., 2000), como broncodilatador no tratamento da asma brônquica, congestão nasal, rinite alérgica e sinusite (WHO, 1999) e em suplementos alimentares indicados para perda de peso e aumento de ganho muscular (Greenway, 2001).

$\mathrm{Na}$ Europa, preparações contendo efedra são aprovadas pela Comissão E para o tratamento de problemas respiratórios em adultos e crianças maiores de seis anos, sendo recomendado o uso por apenas um curto período de tempo, devido ao fenômeno de taquifilaxia (perda do efeito após poucas doses) e risco de dependência (Blumenthal, 2000; Blumenthal et al., 2002).

Nos Estados Unidos o Food and Drug Administration (FDA) registrou centenas de reações adversas atribuídas a compostos contendo efedrinas, que variam desde complicações mais leves, como dores de cabeça, palpitação, nervosismo, insônia e irritabilidade, até ataques cardíacos, hipertensão, batimento cardíaco irregular, derrames, problemas psiquiátricos e morte (Caveney et al., 2001; Samenuk, 2002; Berman-Fugh \& Myers, 2004; Bouchard, 2005; AHFS, 2006; Schekelle et al., 2006), principalmente por atuarem sobre os receptores adrenérgicos (Haller et al., 2002; Wooltorton
\& Sibbald, 2002). Após várias tentativas, em abril de 2004, o FDA proibiu a venda produtos contendo efedrinas nos Estados Unidos (Bent et al., 2004).

No Brasil, as formulações a base de efedrina e pseudoefedrina não foram proibidas, mas têm sua venda regulamentada pela Portaria 344/1998 - lista D1, de Substâncias Precursoras de Entorpecentes e/ou Psicotrópicos (Sujeitas a Receita Médica sem Retenção) (Brasil, 2003a). De acordo com a portaria $n^{\circ} 169 / 2003$ do Ministério da Justiça, a atividade de compra, venda, fabricação, transporte e armazenamento de efedrina e pseudoefedrina (lista I) pura ou em misturas, estão sujeitos ao controle pela Polícia Federal e só podem ser comercializados com autorização especial do Ministério da Saúde e em farmácias, com apresentação de receita médica, e nunca em academias ou lojas especializadas (Brasil, 2003b). Entretanto, seguindo uma tendência mundial, o comércio ilegal é freqüente, alimentado pela carência de fiscalização.

Em recente trabalho de revisão, Soni et al. (2004) reportam que as propriedades farmacológicas e toxicológicas de preparações à base de efedra dependem fortemente do teor de efedrinas e das associações apresentadas. Segundo estes autores, estudos clínicos e pré-clínicos, bem como o longo histórico do uso de efedra e seus alcalóides, indicam que seu emprego pode ser seguro se usado segundo as recomendações dos códigos oficiais. Os sérios efeitos adversos observados estariam relacionados, principalmente, ao seu uso excessivo em formulações ditas "suplementos alimentares" devido a associações e interações com outros fármacos ou drogas, susceptibilidade individual, presença de contaminantes, entre outros fatores. Assim, o conhecimento da composição química de espécies de Ephedra e a correta orientação ao público são de fundamental importância para evitar acidentes toxicológicos.

Levando em consideração a importância farmacológica e toxicológica da presença de efedrinas em espécies de efedra e ao fato de haver questionamentos quanto à produção de alcalóides nas espécies americanas, este estudo teve como objetivos avaliar a presença de efedrina em amostras de Ephedra tweediana e E. triandra, coletadas em Porto Alegre/RS, aspecto ainda não relatado na literatura consultada.

\section{MATERIAL E MÉTODOS}

\section{Material vegetal}

O material vegetal foi constituído de partes aéreas de Ephedra tweediana Fisch \& C.A. Meyer coletadas de populações nativas da Reserva Biológica Lami José Lutzenberger, em Porto Alegre/RS, e de partes aéreas de Ephedra triandra Tul., obtidas de plantas cultivadas em Porto Alegre/RS (Bairro Agronomia). Os exemplares das espécies foram identificados por R. Singer e depositadas no Herbário do Departamento 
de Botânica da UFRGS (ICN). Amostras comerciais de efedra foram cedidas por farmácias de manipulação locais e possuem procedências diversas, conforme descrito na Tabela 1.

\section{Preparação da amostra: extração e derivatização}

Para extração de efedrinas de amostras de efedra foi utilizada extração com solvente, metodologia adaptada de Schaneberg et al. (2003). Aproximadamente $500 \mathrm{mg}$ de material vegetal triturado foram colocados em tubo de ensaio cônico de $15 \mathrm{~mL}$ (tipo Falcon), contendo $6 \mathrm{~mL}$ de acetona, sonicados por $15 \mathrm{~min}$ e centrifugados por $10 \mathrm{~min}$ a $3000 \mathrm{rpm}$. O sobrenadante foi retirado e transferido para outro frasco e a extração foi repetida mais 2 vezes, sendo as fases orgânicas reunidas e concentradas a $40{ }^{\circ} \mathrm{C}$ sob atmosfera de nitrogênio. Após remoção completa da acetona, as amostras foram derivatizadas com $100 \mu \mathrm{L}$ de ciclohexanona P.A. (CH) a $100{ }^{\circ} \mathrm{C}$ por 30 min e analisadas por CG/EM.

\section{Análise por cromatografia à gás acoplada a detector de massas (CG/EM)}

As análises por CG/EM foram realizadas em equipamento CG 3800 VARIAN®; injetor 1079 split/ splitless com amostrador automático 8200 VARIAN® acoplado ao detector de massas - massas (SATURNO CG/ MS/MS 2000) VARIAN®. Hélio ultrapuro foi utilizado como gás de arraste com fluxo constante de $1 \mathrm{~mL} / \mathrm{min}$. Para a separação dos constituintes das amostras foi utilizada uma coluna capilar CP-SIL 8CB LOW BLEED/ MS, com $30 \mathrm{~m}$ de comprimento e $0,25 \mathrm{~mm}$ de diâmetro interno, preenchida com polidimetildifenilsiloxano contendo $5 \%$ de grupamentos fenila em filme de 0,25 $\mu \mathrm{m}$. A temperatura da coluna foi programada para 80 ${ }^{\circ} \mathrm{C} / 2 \mathrm{~min}, 50{ }^{\circ} \mathrm{C} / \mathrm{min}$ até $250{ }^{\circ} \mathrm{C} / 2 \mathrm{~min}, 100{ }^{\circ} \mathrm{C} / \mathrm{min}$ até $280^{\circ} \mathrm{C} / 1 \mathrm{~min}$. As temperaturas do injetor e da linha de transferência para o detector de massas foram ajustadas para $220{ }^{\circ} \mathrm{C}$ e $260{ }^{\circ} \mathrm{C}$, respectivamente. $\mathrm{O}$ volume de injeção foi de $1 \mu \mathrm{L}$ no modo split (1:10). Nas condições descritas, o tempo de retenção médio (TR) da efedrina derivatizada com $\mathrm{CH}$ foi de 6,50 min e da pseudoefedrina derivatizada 6,40 min (Figura 1). As análises foram processadas no modo de impacto de elétrons com uma energia de ionização de $70 \mathrm{eV}$. Inicialmente o detector de massas foi ajustado no modo FULL-SCAN, com intervalo de massas de $\mathrm{m} / \mathrm{z} 40$ a 400 u.m.a., sendo monitorados todos os íons obtidos na fragmentação dos analitos. Uma vez ajustada a metodologia analítica, para se obter maior sensibilidade, o detector foi programado para o modo SIM (selected ion monitoring), sendo monitorados os íons de relação de massa/carga $(\mathrm{m} / \mathrm{z}) 58$

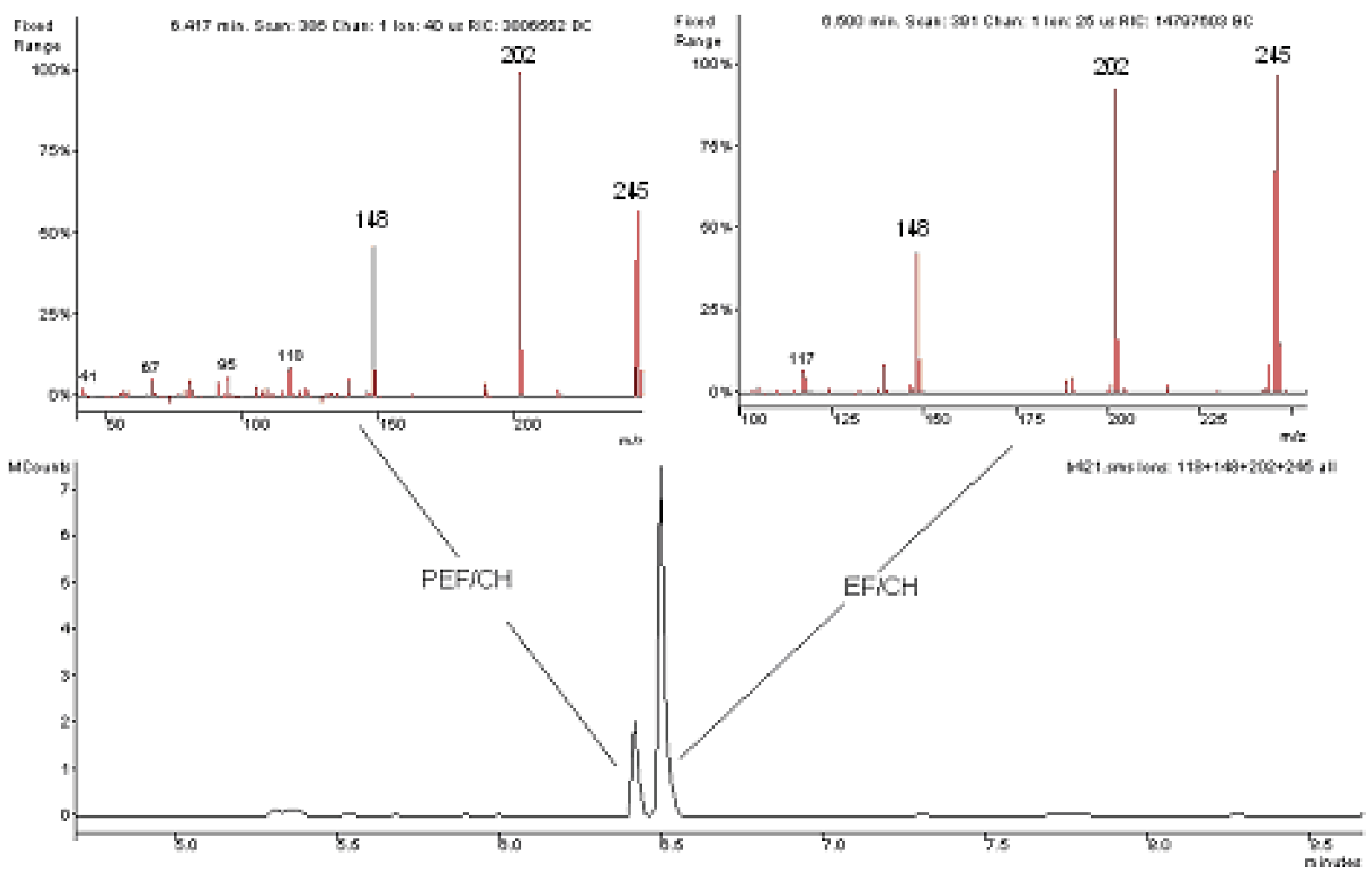

Figura 1. Cromatograma e espectros de massas obtidos por CG/EM, com padrão de efedrina (EF) e pseudoefedrina (PEF), após derivatização com ciclohexanona $(\mathrm{CH})$, utilizando coluna capilar CP-SIL 8CB LOW BLEED/MS. 

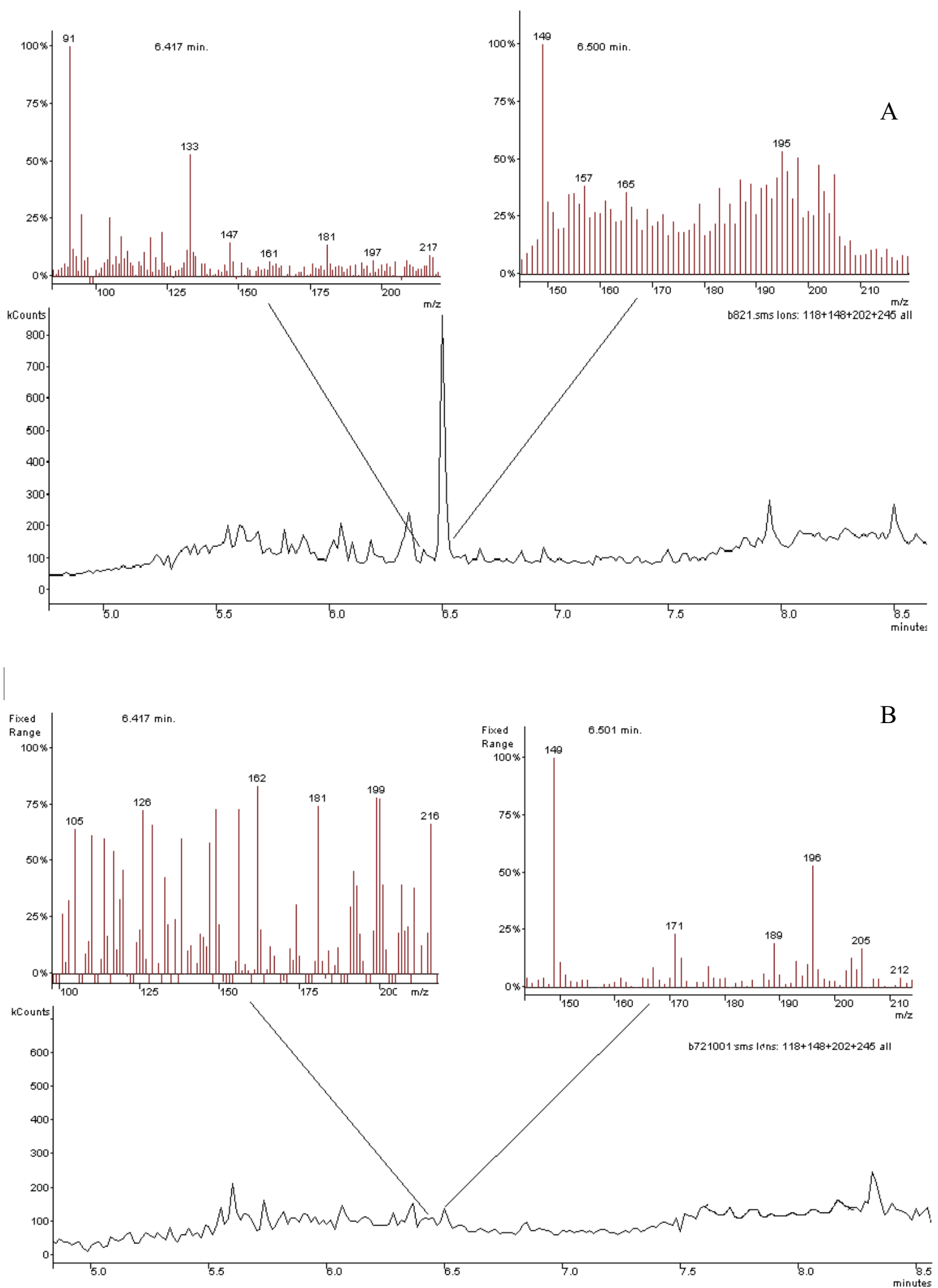

Figura 2. Cromatogramas e espectros de massas, obtidos por CG/EM, com amostras de Ephedra tweediana (A) e E. triandra (B), indicando ausência de efedrina (TR 6,5 min) e pseudoefedrina (TR 6,4 min), sendo utilizada coluna capilar CP-SIL 8CB LOW BLEED/MS. Os picos cromatográficos em 6,5 min e 6,4 min apresentam espectros de massas diferentes dos espectros da efedrina e pseudoefedrina. 


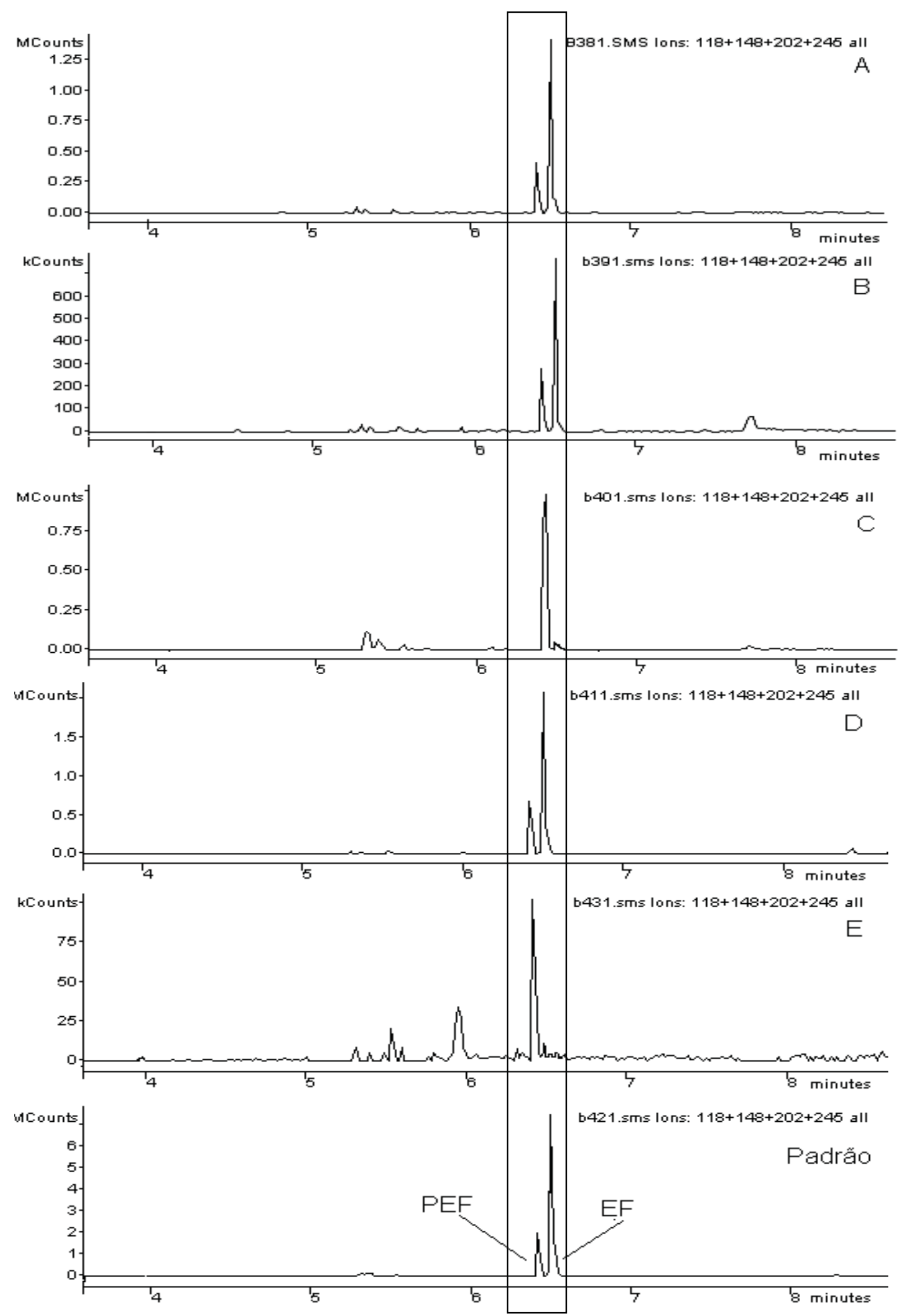

Figura 3. Cromatogramas obtidos com amostras comerciais de efedra (amostras A a E) e padrão de efedrina (EF) e pseudoefedrina (PEF) por CG/EM utilizando coluna capilar CP-SIL 8CB LOW BLEED/MS. 
Tabela 1. Amostras comerciais de efedra de origem asiática, com seus respectivos teores* de efedrina (EF) e peudoefedrina (PEF).

\begin{tabular}{|c|c|c|c|c|c|c|}
\hline Amostra & $\begin{array}{c}\text { Espécie } \\
\text { declarada }\end{array}$ & Procedência & $\begin{array}{l}\text { Prazo de } \\
\text { validade }\end{array}$ & $\begin{array}{c}\text { Teor } \\
\text { PEF / EF } \\
(\%)\end{array}$ & $\begin{array}{c}\text { Relação } \\
\text { PEF e EF (\%) }\end{array}$ & $\begin{array}{c}\text { Efedrinas } \\
\text { Totais } \\
\text { PEF + EF }(\%)\end{array}$ \\
\hline $\mathrm{A}$ & E. distachya & Paquistão & $01 / 2008$ & $0,06 / 0,29$ & $17,14 / 82,86$ & 0,35 \\
\hline $\mathrm{B}$ & E. sinica & Paquistão & $03 / 2007$ & $0,07 / 0,19$ & $26,90 / 73,10$ & 0,26 \\
\hline $\mathrm{C}$ & E. distachya & Índia & $11 / 2005$ & $0,22 / 0,00$ & $100,00 / 0,00$ & 0,22 \\
\hline $\mathrm{D}$ & Ma Huang & Índia & $03 / 2009$ & $0,24 / 0,41$ & $36,92 / 63,08$ & 0,65 \\
\hline $\mathrm{E}$ & Ma Huang & Índia & $11 / 2005$ & $0,06 / 0,00$ & $100,00 / 0,00$ & 0,06 \\
\hline
\end{tabular}

* Determinados por CG/EM, modo SIM (selected ion monitoring), pela técnica de padronização externa.

e 77 para efedrina sem derivatizar; e os íons $\mathrm{m} / \mathrm{z} 202$, 148, 118 e 245 para EF e PEF derivatizadas (Figura 1) (Sebben et al., 2006). Foram utilizados padrões de efedrina e pseudoefedrina na forma de cloridrato, base anidra, e os valores foram corrigidos através de fator de correção.

\section{RESULTADOS E DISCUSSÃO}

Extratos oriundos de Ephedra tweediana e E. triandra, coletadas em Porto Alegre/RS, foram avaliados quanto a presença de efedrina (EF) e pseudoefedrina (PEF) demonstrando ausência destes alcalóides. Entretanto, como pode ser visualizado na Figura 2, foi caracterizada a presença de 2 picos cromatográficos nos tempos de retenção da EF e PEF, os quais apresentaram espectros de massas diferentes daqueles obtidos com os espectros dos padrões (Figura 1), evidenciando não se tratarem destas substâncias. A presença destes picos pode justificar os relatos na literatura sobre presença de efedrinas em espécies americanas, pois uma vez que a análise seja realizada sem utilizar o detector de massas, resultados equivocados podem ser gerados.

Quando o mesmo método foi utilizado para análise de amostras comerciais de efedra (Tabela 1), estas apresentaram picos cromatográficos nos mesmos tempos de retenção esperados para a EF e/ou PEF (Figura 3) com espectros de massas característicos (Figura 1), indicando a presença destes alcalóides nas amostras comerciais. Os resultados obtidos demonstram teores entre 0,06 a $0,65 \%$ de efedrinas $(\mathrm{PEF}+\mathrm{EF})$ nas amostras analisadas. Cabe salientar que as amostra $\mathrm{C} \mathrm{e}$ $\mathrm{E}$, que apresentaram os menores teores, estavam com o prazo de validade vencido. Os teores de efedrinas encontrados nas amostras comerciais estão dentro do relatado na literatura (Schaneberg et al., 2003) e abaixo do preconizado pelos códigos oficiais (JP XIII, 1996).

Muitos métodos têm sido relatados na literatura científica para detecção de efedrina em extratos vegetais, suplementos alimentares e amostras biológicas, utilizando as técnicas de cromatografia à líquido de alta eficiência (JP XIII, 1996; Gurley et al., 1998; Gmeiner et al., 2002), cromatografia à gas (Garcia et al., 2005; Marchei et al., 2006), eletroforese capilar (Mateus-
Avois et al., 2003) e espectrometria de massas (Gay et al., 2001; Spyridaki et al., 2001; Tseng et al., 2003). Entretanto, a maioria destes métodos está relacionada à análise de efedrinas em urina ou suplementos alimentares, reportando extensas fases de preparação da amostra e não permitindo eficiente separação de EF e PEF (Spyridaki et al., 2001; Tseng et al., 2003), o que é de extrema importância em se tratando de análises toxicológicas, dopagem e monitoramento terapêutico, uma vez que ambos isômeros apresentam diferentes atividades farmacológicas e ações sobre o SNC, considerando que metodologias envolvendo detector de massas são preferidas em alto grau para identificação de substâncias contidas em produtos de origem desconhecida, esta foi à metodologia escolhida para a realização deste trabalho.

Em estudos preliminares desenvolvidos por nosso grupo de pesquisa (Sebben et al., 2006), muitas das metodologias que empregam CG/EM descritas na literatura foram reconduzidas e os resultados obtidos, para análise de efedrinas em urina, demonstraram variabilidade muito grande, em termos qualitativos e quantitativos, caracterizando a necessidade de ajustes do método em todas as suas etapas, para obtenção de resultados confiáveis, o que foi realizado previamente ao desenvolvimento deste estudo.

$\mathrm{Na}$ etapa de preparação da amostra, após a extração das efedrinas a partir de extratos vegetais, para aumentar a sensibilidade, melhorar o desempenho da análise cromatográfica, estabilizar os analitos e evitar a perda por adsorção na fase estacionária da coluna cromatográfica ou por decomposição térmica, durante as análises nos módulos CG e EM, faz-se necessária a derivatização da amostra. A metodologia utilizada neste trabalho e consiste no emprego de $\mathrm{CH}$ como agente de derivatização, a qual, além de apresentar baixo custo, facilidade de execução da técnica, relativa segurança ao manipulador e melhor sensibilidade, permite a separação da EF e PEF utilizando colunas de CG normais, a base de sílica, melhor linearidade e maiores limites de detecção e quantificação, resultados não alcançados quando utilizada efedrina não derivatizada (Sebben et al., 2006).

Outro aspecto a ser considerado é que no modo 
impacto de elétrons os fragmentos característicos da EF não derivatizada são m/z 58 e 77, fragmentos de baixa massa molecular e inespecíficos, podendo ser confundidos com uma gama de interferentes. $\mathrm{O}$ aumento da massa molecular (MM 245) com a reação entre a $\mathrm{EF}$ e a $\mathrm{CH}$ produz íons de relação $\mathrm{m} / \mathrm{z}$ maiores e mais específicos (m/z 202, 148, 118), melhorando a resposta cromatográfica.

\section{CONCLUSÕES}

Os resultados obtidos neste trabalho permitiram confirmar a presença de efedrina (EF) e/ ou pseudoefedrina (PEF) em amostras comerciais de efedras asiáticas e assegurar a ausência destes alcalóides em Ephedra tweediana e E. triandra, coletadas em Porto Alegre, ratificando a hipótese de que as efedras americanas não produzam alcalóides.

É importante salientar que os resultados obtidos em estudos filogenéticos recentes sustentam a monofilia de um clado americano de Ephedra, que contêm as espécies sul e norte-americanas (Huang et al., 2005, Ickert-Bond \& Wojciechowski, 2004, Rydin et al., 2004). Por sua vez, dentro deste clado, as espécies sul-americanas se agrupam em um clado menor. Em outras palavras, E. tweediana e E. triandra (espécies sem efedrinas) são filogenéticamente próximas. Espécies que sabidamente contêm efedrinas, tais como E. sinica, E. intermedia e E. equisetina pertencem a dois clados asiáticos de Ephedra, não relacionados com as espécies do continente americano. É necessário fazer uma amostragem mais abrangente em relação à presença/ausência de efedrinas no gênero como um todo, de modo a poder inferir com segurança como foi o histórico evolutivo deste caráter. Contudo, com os dados atuais é possível levantar a hipótese de que a ausência de efedrinas seja uma condição derivada nas espécies americanas; pois estes alcalóides aparecem em pelo menos dois clados asiáticos do gênero.

\section{AGRADECIMENTOS}

Os autores agradecem ao Centro de Informação Toxicológica do RS pelo apoio laboratorial, à Bióloga Patrícia B. R. Witt, responsável técnica e gerente da Unidade de Conservação da Reserva Biológica do Lami, pelo fornecimento de amostras de E. tweediana, e às Farmácias locais por forneceram as Ephedras comerciais.

\section{REFERENCIAS}

ABC 2002. The ABC Clinical Guide to Herbs. American Botanical Council, 2002. www.herbalgram.org, acessada em 20 de agosto de 2006.

AHFS 2006. American Hospital Formulary Service. www. ashp.org/ahfs, acessada em 03 setembro de 2006.
Barbosa-Filho JM, Piuvezam MR, Moura MD, Silva MS, Lima KVB, Cunha EVL, Fechine IM, Takemura OS 2006. Anti-inflammatory activity of alkaloids: A twentycentury review. Rev Bras Farmacogn 16: 109-139.

Bent S, Padula A, Neuhaus J 2004. Safety and efficacy of Citrus aurantium for weight loss. Am J Cardiol 94: 1359-1361.

Berman-Fugh A, Myers A2004. Citrus aurantium, an ingredient of dietary supplements marketed for weight loss: current status of clinical and basic research. Exp Biol Med 229: 698-704.

Blumenthal M, Goldberg A, Brinckman J 2000. Herbal Medicine - Expanded Comission E Monographs. Newton: American Botanical Council.

Blumenthal M, Goldberg A, Brinckman J 2002. Ephedra. Herbal Medicine. In Expanded Comission E Monographs. Texas: American Botanical Council.

Bouchard NC 2005. Ischemic stroke associated with use of an Ephedra-free dietary supplement containing synephrine. Mayo Clin Proc 80: 541-545.

Brasil 2003a. Ministério da Saúde. Agencia Nacional de Vigilância Sanitária (ANVISA). Resolução RDC $\mathrm{n}^{\circ}$ 18. Diário Oficial da União, Brasília, DF, de 28 de janeiro.

Brasil 2003b. Ministro da Justiça. Portaria $n^{\circ} 169$. Diário Oficial da União, Brasília, DF, de 21 de fevereiro.

Bruneton J 1992. Elementos de Fitoquimica y de Farmacognosia. Zaragoza: Acribia.

Caveney S, Charlet DA, Freitag H, Maier-Stolte M, Strarratt AN 2001. New observations of the secondary chemistry of world Ephedra (Ephedraceae). Am J Bot 88: 1199-1208.

Foster S, Tyler VE 1999. Tyler's Honest Herbal. A sensible guide to use of herbs and related remedies. New York: Haworth Herbal Press.

Garcia PR, Yonamine M, Moreau RLM 2005. Determinação de efedrinas em urina por cromatografia em fase gasosa (CG/DNP) para o controle da dopagem no esporte. Rev Bras Cienc Farm 41: 351-358.

Gay ML, White KD, Obermeyer WR, Betz JM, Musser SM 2001. Determination of ephedrine-type alkaloids in dietary supplements by LC-MS using stable-isotope labeled internal standard. $J A O A C$ Int 84: 761-769.

Gmeiner G, Geisendorf T, Kainzbauer J, Nikolajevic M, Tausch H 2002. Quantification of ephedrines in urine by column-switching high-performance liquid chromatography. $J$ Chromatogr B 768: 215-221.

Greenway FL 2001. The safety and efficacy of pharmaceutical and herbal caffeine and ephedrine use as a weight loss agent. Obes Rev 2: 199-211.

Gurley BJ, Wang P, Gardner SF 1998. Ephedrine-type alkaloid content of nutritional supplements containing Ephedra sinica (Ma huang) as determined by high performance liquid chromatography. J Pharm Sci 87: 1547-1553.

Haller CA, Jacob III P, Benowitz NL 2002. Pharmacology of ephedra alkaloids and caffeine after single-dose dietary supplement use. Clin Pharmacol Ther 71: 421-432.

Huang J, Gianassi DE, Price RA 2005. Phylogenetic relationships in Ephedra (Ephedraceae) inferred from chloroplast and nuclear DNA sequences. $\mathrm{Mol}$ Phylogenet Evol 35: 48-59. 
Hunziker JH 1995. Flora Fanerogámica Argentina. Fascículo 4: Ephedraceae. Pró-Flora, CONICET: 15-23.

Ickert-Bond SM, Wojciechowski MF 2004. Phylogenetic relationships in Ephedra (Gnetales): Evidence from nuclear and chloroplast DNA sequence data Syst Bot 29: 834-849.

JP XIII 1996. The Japanese Pharmacopoeia. 13ª Ed. Tokyo: The Spciety of Japanese Pharmacopeia.

Konno C, Tagushi T, Tamada M, Hikino H 1979. Ephedroxane, anti-inflammatory principle of Ephedra herbs. Phytochemistry 18: 697-698.

Marchei E, Pellegrini M, Pacifici R, Zuccaro P, Pichini S 2006. A rapid and simple procedure for the determination of ephedrine alkaloids in dietary supplements by gas chromatography-mass spectrometry. J Pharm Biomed 41: 1633-1641.

Mateus-Avois L, Mangin P, Saygy M 2003. Development and validation of a capillary zone electrophoresis method for the determination of ephedrine and related compounds in urine without extraction. $J$ Chromatogr B 791: 203-216.

PPRC 1997. Pharmacopeia of the People's Republic of China 1997 Beijing, China: Chemical Industry Press.

Rydin C, Pedersen KR, Friis EM 2004. On the evolutionary history of Ephedra: Cretaceous fossils and extant molecules. Proc Nat Acad Sci USA 101: 1657116576.

Samenuk D 2002. Adverse cardiovascular events temporally associated with ma huang, an herbal source of ephedrine. Mayo Clin Proc 77: 12-16.

Schaneberg BT, Crockett S, Bedir E, Khan IA 2003. The role of chemical fingerprinting: application to Ephedra. Phytochemistry 62: 911-918.

Schekelle P, Morton S, Maglione M 2006. Ephedra and ephedrine for weight loss and atletic performance enhancement: clinical efficacy and side effects. www. fda.gov/ohrms/dockets/98fr/95n-0304-bkg-ref-0701-index.htm, acessada em 01 de junho de 2006.

Sebben VC, Paliosa PK, Limberger RP 2006. Efeito dos agentes derivatizantes sobre a efedrina. In: II SIMCRO - Simpósio de Cromatografia, São Pedro, São Paulo.

Soni MG, Carabin IG, Griffiths JC, Burdock GA 2004. Safety of ephedra: lessons learned. Toxicol Lett 150: $97-$ 111.

Spyridaki MHE, Tsitsimpikou CJ, Siskos PA, Georgakopolous CG 2001. Determination of ephedrines in urine by gas chromatography - mass spectrometry. $J$ Chromatogr $B$ 758: 311-314.

Tseng YL, Hsu HR, Kuo FH, Shieh MH, Chang CF 2003. Ephedrines in over-the-counter cold medicines and urine specimens collected during sport competitions. J Anal Toxicol 27: 359-365.

Tyler VE 1993. The honest herbal: a sensible guide to the use of herbs and related remedies. New York: Pharmaceutical Products Press.

Willaman JJ, Scheubert BG 1961. Alkaloid-bearing plants and their contained alkaloids. Agricultural Research Service. United States Department of Agriculture, Washington, Technical Bulletin 1234.

Wink M 1998. A short history of alkaloids. In: Roberts MF, Wink M Ed. Alkaloids: Biochemistry, Ecology and Medicinal Applications, New York: Plenum.
Wooltorton E, Sibbald B 2002. Ephedra/ephedrine: cardiovascular and CNS effects. Can Med Assoc J 165: 633 .

WHO 1999. World Health Organization. Regional Office for the Western Pacific. Guidelines for Evaluating the Safety and Efficacy of Herbal Medicines. Manila. 